Article

\title{
CsICE1 Functions in Cold Tolerance by Regulating Polyamine levels May through Interacting with Arginine Decarboxylase in the Tea Tree
}

\author{
Xujun Zhu®, Xue Zhao, Taiyu Ren, Yuanchun Ma, Yuhua Wang and Wanping Fang * \\ College of Horticulture, Nanjing Agricultural University, No.1 Weigang, Nanjing 210095, Jiangsu, China; \\ zhuxujun@njau.edu.cn (X.Z.); 2018804242@njau.edu.cn (X.Z.); 2018104087@njau.edu.cn (T.R.); \\ myc@njau.edu.cn (Y.M.); wangyuhua@njau.edu.cn (Y.W.) \\ * Correspondence: fangwp@njau.edu.cn; Tel./Fax: +86-25-8439-5182
}

Received: 9 April 2020; Accepted: 26 May 2020; Published: 3 June 2020

check for updates

\begin{abstract}
Background: The identification of C-repeat binding factor (CBF), and the characterization as an inducer of CBF Expression 1 (ICE1), and a major activator for C-repeat binding factor, were important breakthroughs in the cold signaling network. Methods: In the present study, the full length cDNA of ICE1 was isolated from the tea tree (Camellia sinensis). CsICE1 protein was located in the cell nucleus as revealed by subcellular localization analysis. To investigate the biological functions of CSICE1, a transgenic line fused with the CSICE1 gene in Arabidopsis thaliana (arabidopsis) was generated by the floral dip method. Results: The CsICE1 was expressed differentially in various tea tree tissues, mostly in buds and leaves, and the transcript level of CSICE1 was increased after $1 \mathrm{~h}$ and peaked at $2 \mathrm{~h}$ under cold treatment. Transcription activity assay indicated that the spermine synthase (SPMS) and arginine decarboxylase (ADC) genes were possible targets of CsICE1. In addition, the values of net photosynthetic rate, transpiration rate, stomatal conductance in transgenic lines declined by less extent than wild-type plants under low temperatures. Furthermore, transcript levels of $A D C$ genes in the transgenic lines had no apparent alteration under normal growth conditions but substantially increased under cold conditions, consistent of changes in free polyamine levels. Taken together, these results demonstrated that CsICE1 plays a positive role in cold tolerance, which may be due to the modulation of polyamine levels through interacting with CSADC.
\end{abstract}

Keywords: biological function; Camellia sinensis; expression analysis; gene cloning; transgenic plant; arginine decarboxylase

\section{Introduction}

Low temperatures adversely affect plant quality, productivity and function as a determinant of geographical distribution and growth [1]. Tea tree (Camellia sinensis) is an important economic crop in China and is often damaged by cold stress, which seriously affects the quality and yield of tea. Cold tolerance has been defined as the ability to emerge and grow normally after planting in cold (but above freezing) soils [2]. Therefore, the improvement of cold tolerance is an important task in tea tree breeding. Genes are highly induced by environmental stress and may have critical functions in directly protecting plants against stresses or in further regulating the expressions of other target genes [3].

The identification of C-repeat binding factor (CBF) [4,5], and the characterization of ICE1 [6] were important breakthroughs in the cold signaling network. The ICE1-CBF-cold regulated (COR) cold response pathway was one of the dominant cold signaling mechanisms mediating cold response $[7,8]$. Cold stress modifies either ICE or a related protein bound to ICE to regulate CBF gene expression. The CBF proteins may be synthesized and banded to the CRT/Dehydration-responsive elements 
(DRE) in the promoter regions of the COR genes to activate their expression, such as COR15a and COR78 [9]. In addition, ICE1 encodes an MYC-type basic helix-loop-helix (bHLH) transcription factor in arabidopsis [10]. To date, ICE1 and ICE2 (an ICE1 homolog) have been reported in various plants, such as apple [11], tomato [12], trifoliate orange [13], and banana [14]. Huang et al. [13] reported that ICE1 of Poncirous trifoliate plays a positive role in cold tolerance by modulation of polyamine levels via interacting with the $A D C$ gene. Therefore, it is reasonable to hypothesize that some undetermined molecular mechanisms associated with polyamine metabolism might also contribute to the cold tolerance imparted by CsICE1.

Tea trees entering a dormancy period are able to survive during winter, as are other woody species. However, early spring frost often occurs and affects the development of tea sprouts, which can cause tea production loss in many temperate regions. To date, some COR genes have been isolated from tea trees [15-18], but little is known concerning the regulatory mechanisms for ICE1-CBF-COR cascade under cold stress. Wang et al. [19] isolated the full length cDNAs of CsICE1 and CsCBF1 and demonstrated that CsCBF1 protein had the ability to bind with the CRT/DRE cis-element from arabidopsis RD29A promoter by electrophoretic mobility shift assay. Ding et al. [20] investigated the pattern of CSICE1 expression under cold temperature $\left(4^{\circ} \mathrm{C},-5^{\circ} \mathrm{C}\right)$, drought, and plant hormone (abscisic acid, ABA; brassinolide, BR) treatment by real-time quantitative PCR. In our present study, we further report that overexpression of CsICE1 led to enhanced cold tolerance in arabidopsis transgenic plants. Transcription activity assay indicated that the CSSPMS and CSADC genes were possible targets of CsICE1. We also demonstrated that transcript levels of ADC genes were elevated in the transgenic plants under cold treatment, concomitant with an increase in free polyamine levels compared with the wild type.

\section{Materials and Methods}

\subsection{Plant Materials and Stress Treatment}

One-year-old tea trees (Camellia sinensis cv. Yingshuang), around $20 \mathrm{~cm}$ tall, were transplanted from a botanic garden in Nanjing Agricultural University (Nanjing, China). The tea trees were then grown in a growth chamber for two weeks with a photoperiod regime consisting of $12 \mathrm{~h}$ light $\left(25^{\circ} \mathrm{C}\right.$, $200 \mathrm{~mol} \cdot \mathrm{m}^{-2} \cdot \mathrm{s}^{-1}, 70 \%$ humidity $) / 12 \mathrm{~h}$ dark $\left(22^{\circ} \mathrm{C}, 70 \%\right.$ humidity $)$. The tea trees were exposed to different treatments, as described next.

For cold treatment, the plants were grown in the soil (water content $16.5 \%$ ) in a growth chamber, which has four layers of partitions, set at $4{ }^{\circ} \mathrm{C}$ for $72 \mathrm{~h}$; the leaves were collected at $0,1,2,4,8,24$, and $72 \mathrm{~h}$ after the treatment. For dehydration treatment, the leaves were placed in empty flasks for $24 \mathrm{~h}$ and re-watering for 7 days; leaves were collected at $0,2,4,8,24$, and $3 \mathrm{~d}, 7 \mathrm{~d}$ after treatment. For salt stress, the plants were placed into flasks containing $150 \mathrm{mM} \mathrm{NaCl}$; the leaves were collected at $0,1,2,4$, 8,24 , and $72 \mathrm{~h}$ after treatment. The samples (one bud and two leaves) were then frozen immediately in liquid nitrogen and stored at $-80^{\circ} \mathrm{C}$ for further analysis.

\subsection{Isolation of the CSICE1 and Bioinformatics Analysis}

Total RNA from the plant leaves was isolated using an RNAprep pure plant kit (Tiangen, Beijing, China). The first strand cDNA was synthesized from $2.0 \mu \mathrm{g}$ DNase I treated RNA using M-MLV reverse transcriptase (Promega, Madison, WI, USA). CsICE1 gene was amplified according to the sequence (NCBI accession no.: GQ229032.2) by PCR using the primers listed in Table 1. The DNA and protein sequences were characterized using DNAMAN software. Clustal X (University College Dublin, Dublin, Ireland) was employed to perform multiply alignments. 
Table 1. Primers used in the study of gene expression analysis.

\begin{tabular}{ccc}
\hline Primers & Sequence $\mathbf{( 5}^{\prime} \mathbf{\prime}^{\prime} \mathbf{\prime}^{\prime}$ & Remarks \\
\hline Cs $\beta$-actin-F & GCCATCTTTGATTGGAATGG & Internal reference \\
Cs $\beta$-actin-R & GGTGCCACAACCTTGATCTT & Internal reference \\
CsICE1-FL-F & ATGGAAGTGAGTGAAGTGAATGAG & ORF amplification \\
CsICE1-FL-R & AAGAAACCTACATCATGAAGCCAGC & ORF amplification \\
CsICE1-gfp-F & GCTCTAGAATGGAAGTGAGTGAAGTGAATGAG & Subcellular localization \\
CsICE1-gfp-R & CGGGATCCAAGAAACCTACATCATGAAGCCAGC & Subcellular localization \\
CsICE1-Q-F & ATGTTTTGTAGCCGCAGAC & qPCR \\
CsICE1-Q-R & GCTTTGATTTGGTCAGGATG & qPCR \\
AtADC1-Q-F & accgtacgattgattacga & qPCR \\
AtADC1-Q-R & taacttaaaactttaaaac & qPCR \\
AtADC2-Q-F & atcgtaaaatcgtcattgta & qPCR \\
AtADC2-Q-R & tcatggatctcatcatgcct & qPCR \\
\hline
\end{tabular}

\subsection{Subcellular Localization of CsICE1}

The CSICE1 open reading frame (ORF) without a stop codon was amplified by PCR with primers listed in Table 1. The PCR product was digested and then cloned into a pJIT166-GFP vector. Subsequently, particle bombardment (PDS-1000/He biolistic particle delivery system, Bio-Rad Laboratories) was conducted by transiently expressing the green fluorescence protein (GFP) fusion vector (CsICE1-GFP) and control vector (GFP) in onion epidermal cells [21]. After incubation at $25^{\circ} \mathrm{C}$ for $16 \mathrm{~h}$ in the dark, the epidermal layers were peeled off. Finally, a confocal laser microscope (LSCM, TCS SP2, Leica, Heidelberg, Germany) was used to observe the localization of the CsICE1 protein.

\subsection{Expression Analysis by Quantitative Real-Time RT-PCR}

Quantitative real-time RT-PCR was performed to evaluate the expression levels of CsICE1 under different stress treatments and in different tissues. Each $20 \mu \mathrm{L}$ of the PCR reaction solution contained $10 \mu \mathrm{L}$ of $2 \times$ SYBR Premix Ex Taq (TaKaRa, Kyoto, Japan), $10 \mathrm{ng}$ of diluted cDNA, and $0.2 \mu \mathrm{M}$ of gene-specific primers (Table 1 ). The amplification conditions of thermocycling were listed as follows: $95^{\circ} \mathrm{C}$ for $30 \mathrm{~s}$ and 40 cycles of $95^{\circ} \mathrm{C}$ for $5 \mathrm{~s}$ and $60^{\circ} \mathrm{C}$ for $30 \mathrm{~s}$. The Camellia sinensis-actin gene (NCBI accession no.: HQ420251) was used as the reference gene. Each sample was analyzed in four biological replicates, and the relative expression levels were calculated according to the $2^{-\Delta \Delta C T}$ method.

\subsection{Generation and Characterization of Transgenic Plants}

The full length CSICE1 gene was amplified, digested, and subsequently recombined into the pCAMBIA1301 vector with a modified cauliflower mosaic virus (CaMV) 35S promoter. The CsICE1 gene was introduced to wild-type (WT) arabidopsis (Columbia ecotype) by using a floral dip method [22]. Positive transformants were screened in an MS medium containing $50 \mathrm{mg} \cdot \mathrm{L}^{-1}$ hygromycin, and the integration of transgene in arabidopsis was confirmed by reverse transcription-polymerase chain reaction (RT-PCR). The expression of the CsICE1 gene from positive transformants was determined by quantitative RT-PCR.

\subsection{Determination of Polyamines Levels}

The procedures for extraction of free polyamines, and for high-performance liquid chromatography (HPLC) analysis, were described by Zhu et al. (2015). Free polyamines were extracted by $5 \%$ cold perchloric acid and derivatized by benzoyl chloride. Polyamines were separated by an HPLC (Shimadzu, Japan) equipped with a $C_{18}$ reverse-phase column $(4.6 \times 250 \mathrm{~mm}$, particle size $5.0 \mu \mathrm{m})$ with programmed gradient solvents (acetonitrile/water) (Zhu et al., 2015). 


\subsection{Cold Stress Assay of the Transgenic Plants}

Transgenic plants were treated with cold at either chilling $\left(4^{\circ} \mathrm{C}\right)$ or freezing $\left(0^{\circ} \mathrm{C}\right)$ temperatures. For chilling treatment, transgenic plant seedlings grown at $22^{\circ} \mathrm{C}$ were kept at $4{ }^{\circ} \mathrm{C}$ for $48 \mathrm{~h}$ or $5 \mathrm{~d}$. For freezing stress, 35-d-old plants were first treated at $0{ }^{\circ} \mathrm{C}$ for $24 \mathrm{~h}$ and then moved to an ambient environment for further growth for $5 \mathrm{~d}$ (recovery), followed by an assessment of survival rate. Leaves collected before and after the freezing treatment were used for the analysis of polyamine levels, ROS accumulation, and expression of $A D C$ (AtADC1 and AtADC2) genes.

\subsection{Transactivation Activity Assay}

Transactivation activity assay was performed as described previously [21]. In brief, $1.2 \mu \mathrm{g}$ of Luciferase (LUC) construct and $0.4 \mu \mathrm{g}$ of Renilla Luciferase (rLUC) construct were used for each bombardment. The rLUC construct includes the rLUC gene driven by the CaMV $35 S$ promoter. Relative luciferase activity was obtained using the Dual-Luciferase Reporter Assay System (Promega, Madison, WI, USA) and luminescence reader (Lumat LB9507 Berthold, Tokyo, Japan).

\subsection{Physiological Measurements}

Photosynthesis parameters were measured by a portable photosynthesis measurement system LCpro-SD (Analytical Development Co. Ltd, Hoddesdon, UK). Net photosynthetic rate $\left(P_{\mathrm{n}}\right)$, transpiration rate $\left(T_{\mathrm{r}}\right)$, stomatal conductance $\left(S_{\mathrm{c}}\right)$, and water use efficiency (WUE) of leaves were measured using the youngest leaves after cold treatment for $24 \mathrm{~h}$. Photosynthesis was measured twice in each sampled leaf, and samples were triplicated for each treatment [23].

\subsection{Statistical Analysis}

All data were analyzed using SPSS (version 18.0 for Windows; IBM Corp., Armonk, NY). Significance was determined by Duncan's test and ANOVA. A probability level of $5 \%(p \leq 0.05)$ was considered statistically significant.

\section{Results}

\subsection{Isolation and Bioinformatics Analysis of CSICE1}

To obtain the Camellia sinensis ICE1 gene, we searched the NCBI database and isolated a complete ORF of 1557 bp (Genbank accession no.: GQ229032.2). The CsICE1 gene encoded a predicted protein of 518 amino acid residues with a predicted molecular mass of $56.8 \mathrm{kDa}$ and $\mathrm{pI}$ value of 5.88. Alignment of CsICE1 with homologs from seven other plant species showed that the deduced protein CsICE1 contains the conserved bHLH domain of 48 aa (from aa 328 to 375), which composed of a 15 aa basic region and two helices (14 and 12 aa respectively) that were connected by a 7 aa loop. However, they varied drastically among each other in the N-terminal region (Figure 1). The bHLH domain and zipper region (ZIP) are indicated by the red cylinder, the square and circle indicate glutamate and arginine, respectively, and the blue line shows the leucine-zipper region.

\subsection{Subcellular Localization of CsICE1}

Sequence analysis using the WOLFPSORT program showed that the CSICE1 protein contains one nuclear localization signal (aa 264-272, NEKKKRKYS), indicating that it may be localized in the nucleus. Assay results are consistent with the predicted sequence analysis. Onion epidermal cells fused with the CsICE1-GFP showed that the green fluorescence was observed only in the nucleus (Figure 2A), implying that CsICE1 was a nuclear protein. However, the pJIT166-GFP control vector fusion protein was distributed throughout the cells (Figure 2B). 


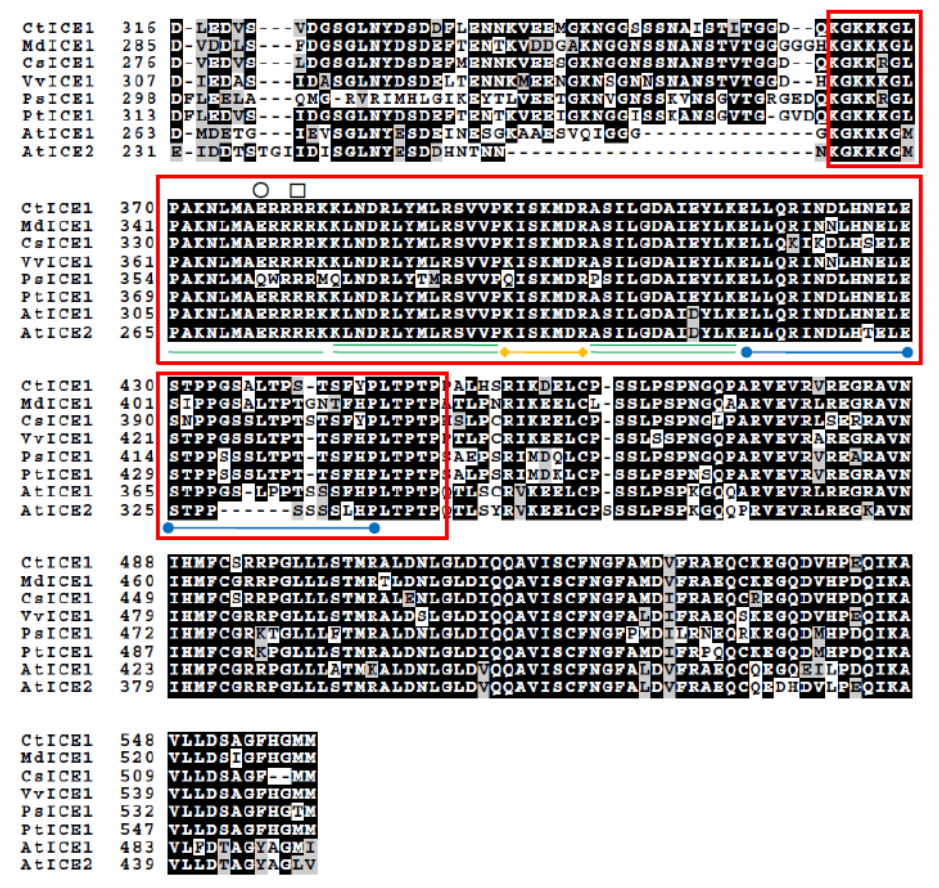

Figure 1. Multiple alignments of the deduced amino acid sequence of CsICE1 and those of other plant species. Short names and accession numbers are as follows: Camellia sinensis (CsICE1, ACT90640.2), Citrius trifoliata (CtICE1, AIZ78178.1), Populus trichocarpa (PtICE1, ABN58427), Vitis vinifera (VvICE1, XP_002284528.1), Populus suaveolens (PsICE1, ABF48720), Arabidopsis thaliana (AtICE1, AAP14668; AtICE2, BAC42644), Malus domestica (MdICE1, ABS50251). The multiple alignment was performed with Clustal $X$ using the default parameters. Identical and similar residues are shown in black and grey backgrounds, respectively. The bHLH domain and zipper region (ZIP) are indicated by the red cylinder, and the square and circle indicate glutamate and arginine, respectively. The single green line below the sequence indicates the basic region, while double green lines represent the helix regions, which are connected by a loop, indicated by the yellow line. The blue line shows the leucine-zipper region.

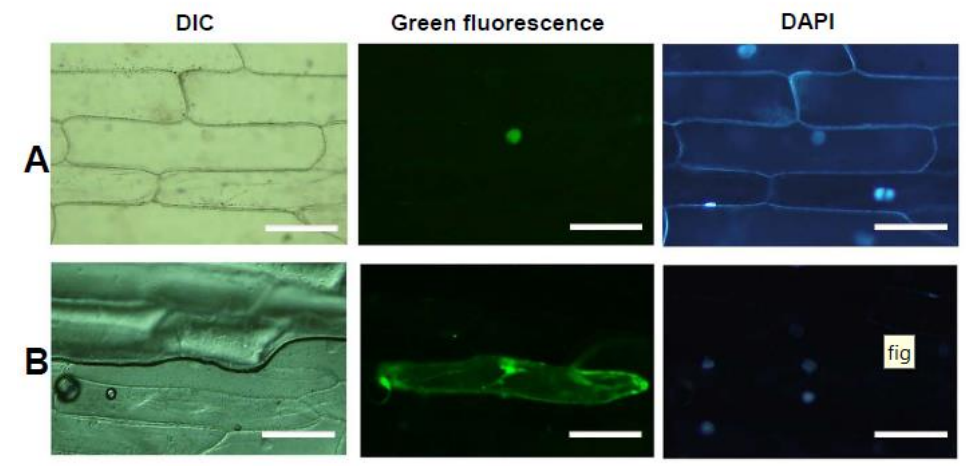

Figure 2. Subcellular localization of CsICE1 protein in onion epidermal cells. Onion epidermal cells were transformed with fusion vector CsICE1-GFP (A) and control vector GFP (B). Images were obtained in bright light to observe the morphological characteristics, in a dark field to detect green fluorescence of the cells, and 4',6-Diamidino-2-Phenylindole, Dihydrochloride (DAPI) staining. Bar $=100 \mu \mathrm{m}$. DIC: differential interference contrast.

\subsection{Tissue-Specific Expression and Stress-Responsive Expression Patterns of CSICE1}

CSICE1 was expressed in roots, stems, leaves, buds, flowers, and fruits under the non-stress condition from Figure 3A. CSICE1 expression was higher in buds and leaves than other tissues, but lowest in roots, stems, and fruits. Hence, CSICE1 was expressed in different tea tree tissues at different expression levels. In order to illustrate the potential biological function of CSICE1, 
the expression profiles of CSICE1 under various stress conditions in leaves were also performed (Figure 3B-D). Under cold treatment, the expression levels of the CSICE1 gene increased drastically and rapidly in response to low temperature and peaked at $2 \mathrm{~h}$, which was 22 -fold greater than that the untreated condition; but the expression levels decreased at $4 \mathrm{~h}$, followed increasing progressively to its highest level at $72 \mathrm{~h}$, whose value was 23 -fold than the initial level (Figure 3B). Under salt treatment, CSICE1 transcripts showed steady elevation with $4 \mathrm{~h}$; but gradually decreased to a lower level at $24 \mathrm{~h}$, followed by an increase at $72 \mathrm{~h}$ (Figure 3C). Under dehydration treatment, the CSICE1 mRNA accumulated slowly and continued to increase to a relatively high level at $4 \mathrm{~h}$, which was 2.2 -fold higher in dehydrate-stressed plants than in non-stress plants; afterward, the expression level decreased at $8 \mathrm{~h}$, followed increasing drastically to its highest level at 3d, which level was 9-fold than the initial level (Figure 3C).
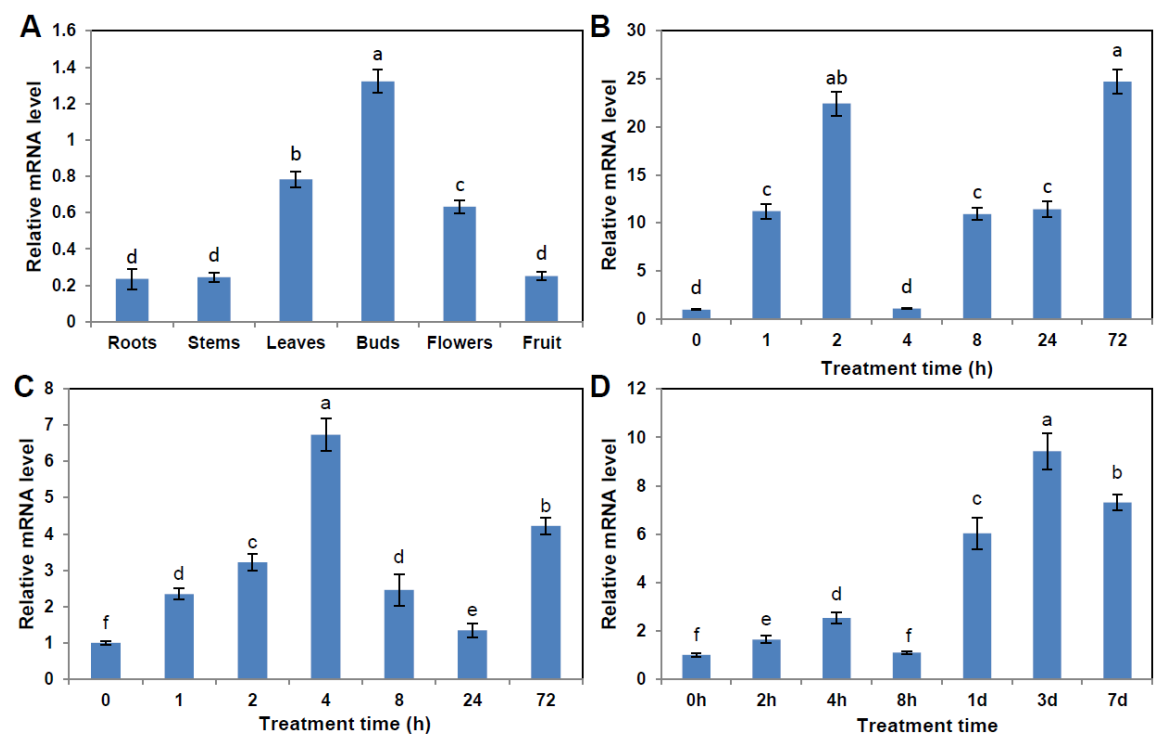

Figure 3. The gene expression analysis of CSICE1 in tea tree. Expression patterns of CSICE1 in different tissues (A), including roots, stems, leaves, buds, flowers, and fruit. Time course expression profiles of CSICE1 in response to cold (B), salt (C), and dehydration (D) stress. Samples were harvested at indicated time intervals. Error bars show the standard deviation based on four replicates. Different letters present a significant difference $(p<0.05)$.

\subsection{Overexpression of CSICE1 Confers Cold tolerance in Transgenic Arabidopsis}

As CsICE1 expression was highly induced at its highest level under cold treatment, efforts were then made to illustrate the biological function in cold tolerance. So, we generated transgenic arabidopsis with recombinant CsICE1 gene induced by a 35S CaMV promoter, and two positive transformants, namely, T2-9, and T3-4, were selected for further tolerance analysis. To assess cold tolerance, transgenic arabidopsis plants were subjected to cold treatment $\left(4^{\circ} \mathrm{C}\right)$. The transgenic and WT plants showed no morphological difference under normal growth condition (Figure $4 \mathrm{~A}, \mathrm{~B}$ ), the leaves of both transgenic and WT plants were green (Figure 4C,D). However, the WT plants suffered more serious cold damage compared to the transgenic plants while under $4{ }^{\circ} \mathrm{C}$ treatment (Figure $4 \mathrm{E}, \mathrm{F}$ ). In addition, the purple color leaves from WT plants were much more than those transgenic lines (Figure 4G,H).

To quantify the photosynthetic and transpiration capacity of transgenic lines under low temperature, the physiological parameters, including the $P_{\mathrm{n}}, T_{\mathrm{r}}, S_{\mathrm{c}}$, and WUE were investigated. The results showed that the values of $P \mathrm{n}, \mathrm{Tr}, S_{\mathrm{c}}$ in both WT and transgenic plants decreased under $3 \mathrm{~d}$ cold treatment (Table 2). However, the values of $P n, T r, S c$ in transgenic lines declined to a lesser extent than WT plants. In addition, WUE showed a similar trend with $P \mathrm{n}, \mathrm{Tr}$, and $\mathrm{Sc}$ under low temperatures. 
Table 2. The effects of cold treatment on the net photosynthetic rate $(P n)$, transpiration rate $(T r)$, stomatal conductance $(S c)$, and water use efficiency (WUE) of WT and transgenic plants. Data were shown as mean $\pm \mathrm{SD}$; different letters presented significant differences among columns $(p<0.05)$. WT presented wild-type plants.

\begin{tabular}{|c|c|c|c|c|}
\hline Plants & $P_{\mathrm{n}}\left(\mu \mathrm{mol} \cdot \mathrm{m}^{-2} \cdot \mathrm{s}^{-1}\right)$ & $T_{\mathrm{r}}\left(\mathrm{mmol} \cdot \mathrm{m}^{-2} \cdot \mathrm{s}^{-1}\right)$ & $S_{\mathrm{c}}\left(\mu \mathrm{mol} \cdot \mathrm{m}^{-2} \cdot \mathrm{s}^{-1}\right)$ & WUE $\left(\mathrm{CO}_{2}\left(\mathrm{mmol} \cdot \mathrm{mol}^{-1} \mathrm{H}_{2} \mathrm{O}\right)\right)$ \\
\hline WT & $5.13 \pm 0.36 \mathrm{~b}$ & $3.47 \pm 0.16 \mathrm{~b}$ & $89.36 \pm 7.43 c$ & $2.08 \pm 0.12 b$ \\
\hline T2-9 & $7.64 \pm 0.31 \mathrm{a}$ & $4.88 \pm 0.17 \mathrm{a}$ & $134.72 \pm 10.35 a$ & $2.19 \pm 0.17 \mathrm{a}$ \\
\hline T3-4 & $7.33 \pm 0.32 a$ & $4.46 \pm 0.12 \mathrm{a}$ & $120.27 \pm 2.12 \mathrm{a}$ & $2.14 \pm 0.12 \mathrm{a}$ \\
\hline
\end{tabular}

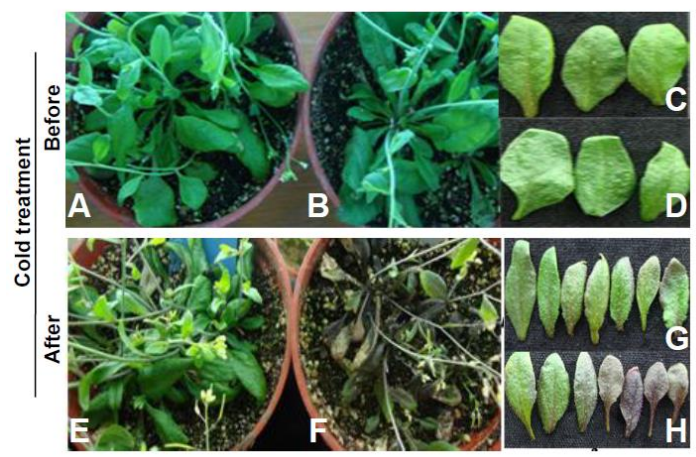

Figure 4. The phenotype of CSICE1 transgenic arabidopsis plants. The plant phenotypes of transgenic $(\mathbf{A}, \mathbf{C}, \mathbf{E}, \mathbf{G})$ and wild type (B,D,F,H) observed before and after cold treatment $\left(4^{\circ} \mathrm{C}\right.$ for 14 days).

\subsection{CSICE1 Interacts With CsADC}

To determine if any protein complex was related to enhancing cold tolerance of transgenic plants overexpressing CSICE1. We tested whether CsICE1 directly transactivates four kinds of stress-responsive genes. Reporter activation occurred when CSADC and CsSPMS were used as effectors (Figure 5), indicating that both $A D C$ and SPMS genes were possible targets of CsICE1. Furthermore, CsADC has been reported previously to play a role in cold tolerance [24], which made us focus on ADC protein. When the CsICE1 (324-518) of amino acids at the C-terminal was deleted, no interaction was detected, which demonstrate that amino acids from positions 324 to 518 in CsICE1 (including the bHLH domain and zipper region) are critical for the transactivation activity of CsICE1.

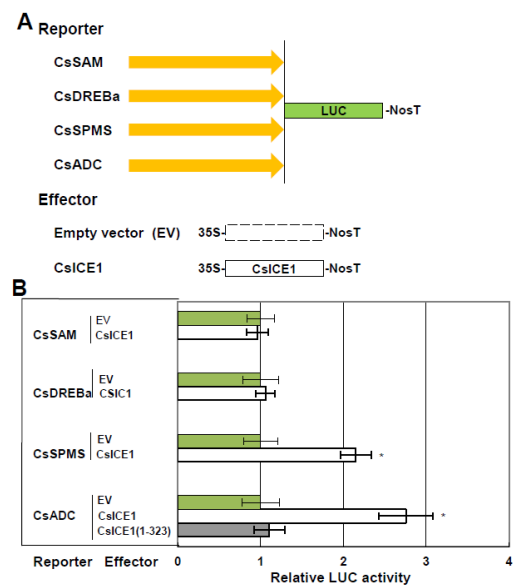

Figure 5. CsICE1 transactivates the ADC gene but not other genes. (A) Two effector constructs and four reporter constructs are schematically illustrated. (B) Transactivation activity assays of CsICE1. Effector, reporter, and Renilla LUC reference plasmids were co-bombarded into arabidopsis mature rosette leaves using a particle delivery system (PDS-1000 He, Bio-Rad, Hercules, CA). After $18 \mathrm{~h}$ of incubation at $23{ }^{\circ} \mathrm{C}$ under darkness, relative luciferase (LUC/rLUC) activities were determined. The asterisk indicates a significant difference $\left({ }^{*} p<0.05\right)$. 


\subsection{Transgenic Arabidopsis Have Higher Level of ADC Transcript and Free Polyamines}

ADC is an enzyme employed for catalyzing arginine to putrescine, which is then converted to spermidine and spermine. The levels of $A D C$ mRNA accumulation and free polyamines were compared between WT arabidopsis and transgenic lines. Expression levels of AtADC1 and AtADC2 were a little higher in both two transgenic arabidopsis lines compared to the WT under non-stress conditions. Exposure to cold stress caused a great induction of AtADC1 and AtADC2 in both two transgenic lines. The transcript levels of AtADC1 in T2-9 and T3-4 were 11.3 and 9.2 times that in the WT (Figure 6A), respectively, while AtADC2 levels in the two transgenic lines were 6.3-8.2 times that in the WT (Figure 6B). Under non-stress condition, the putrescine content in the WT plant was $1.54 \mathrm{~mol} \cdot \mathrm{g}^{-1}$ fresh weight, while T2-9 and T3-4 had putrescine levels of 1.72 and $1.81 \mathrm{~mol} \cdot \mathrm{g}^{-1}$ fresh weight, respectively (Figure 6C,D). Furthermore, the contents of spermidine and spermine in both two transgenic lines were significantly higher than those of the WT. Cold treatment increased the levels of three polyamines in all tested lines, whereas it was noticeable that the polyamine levels in T2-9 and T3-4 were higher than those of the WT.
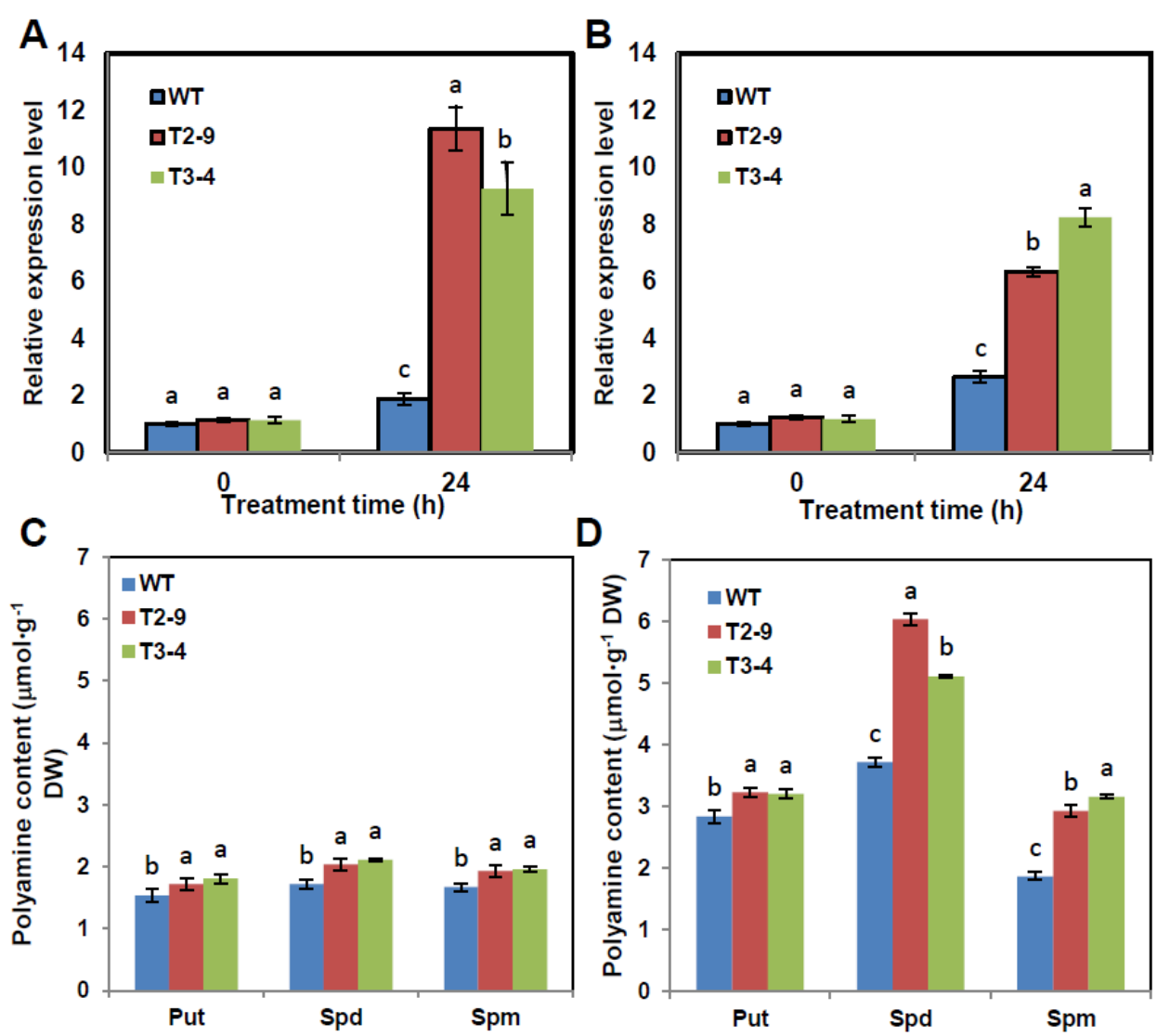

Figure 6. The genes expression analysis of $A D C$ and free polyamine levels in transgenic plants. Expression profiles of $A t A D C 1$ (A) and AtADC2 (B) in WT and transgenic lines exposed to cold stress. Free polyamine levels in WT and transgenic lines before $(\mathbf{C})$ and after $(\mathbf{D})$ cold stress. Different letters present significant difference $(p<0.05)$. WT, wild type; Put, putrescine; Spm, spermine; Spd, spermidine.

\section{Discussion}

The full length cDNA of CSICE1 was isolated from tea tree in this study, the predicted protein contains the conserved bHLH motif (Figure 1). Many reports show that bHLHs play crucial roles in plant responses to various abiotic stresses, including salt [25], drought [26], and cold [10,13]. ICE1 is a well-characterized bHLH protein that acts as an upstream regulator of the transcriptional regulation cascade of the cold response in arabidopsis [27]. However, little is known about the roles of ICE1 
homologs in tea tree, as tea trees prefer warm climate to low temperature. Thus, the characterization of an ICE1 gene of tea tree is important to decipher the cold network pathway pertinent to cold tolerance and to provide valuable gene candidates for genetic manipulation. CSICE1 was induced by abiotic stresses, including cold, salt, and dehydration (Figure 3). The reason for differing observed expression patterns for CSICE1 under cold stress may be due to the individual cold tolerance of tea tree cultivars, which was Yingshuang, Longjing43, and Wuniuzao, respectively $[19,20]$. The strongest induction of CsICE1 transcripts by cold stress forced us to elucidate its function in cold tolerance. The assays demonstrated that overexpression of CSICE1 in arabidopsis resulted in highly enhanced tolerance to cold stresses, indicating that CsICE1 acts as a positive regulator of the cold signaling cascade. On the other hand, overexpression of CsICE1 did not cause negative impacts on plant growth of the transgenic lines under normal growth conditions, suggesting that CsICE1 might have great potential for genetic engineering to improve cold tolerance of tea tree.

In this study, CsICE1 was introduced into arabidopsis to elucidate its biological function. The results showed that CSICE1 overexpressed arabidopsis exhibited dark green leaves, and the WT plants suffered more serious cold damage compared to the transgenic under $4{ }^{\circ} \mathrm{C}$ treatment (Figure 4), presumably, the observation is that WT plants have more purple color leaves than transgenic plants after cold treatment (Figure 4). As well, the higher photosynthetic capacity was found in CsICE1 overexpressed arabidopsis (Table 1). With the temperature decrease, the reduction of photosynthesis in the leaves caused by stomatal closure and reduced water loss by transpiration. It was also suggested that photochemical activity was impaired in plants exposed to cold stress, leading to relatively lower Sc. The non-stomatal limitation of photosynthesis might have occurred in leaves treated with low temperature because of the lower accumulation of biomass. Thus, we suggest that overexpression of CSICE1 can improve the cold tolerance of transgenic plants.

In the present study, CsADC was identified as one of the target genes transactivated by CsICE1, which led us to monitor the expression of $A D C$ genes and endogenous polyamine levels in the transgenic arabidopsis. The expression levels of $A D C$ genes in transgenic lines were only slightly higher than in the WT, under non-stress conditions. However, exposure to the cold temperature resulted in more induction of the $A D C$ genes in the transgenic lines compared with the WT (Figure 6). The greater induction of $A D C$ mRNA was consistent with higher putrescine levels in the transgenic plants compared to the WT. As putrescine is the precursor of spermidine and spermine, active synthesis of putrescine may cause a feed-forward stimulation of downstream metabolism, leading to the promotion of spermidine and spermine synthesis. These results suggest that elevation of polyamine synthesis might contribute to the improvement of cold tolerance in the transgenic lines overexpressing CSICE1. A previous study showed that putrescine accumulation was essential for cold acclimation and plant survival at freezing temperature in plants $[13,28]$. In addition, the increase in endogenous polyamine concentrations by overexpression of polyamine biosynthetic genes or exogenous application of polyamines has been demonstrated to enhance cold tolerance in various plants $[21,29,30]$.

Taking these together, ICE1 of tea tree was highly induced by various abiotic stresses, including cold, salt, and dehydration, with the strongest induction under cold treatment. Overexpression of CSICE1 in arabidopsis conferred enhanced tolerance to low temperatures. In addition, higher levels of $A D C$ transcripts and free polyamines were detected in the transgenic lines. Our results demonstrate that CSICE1 functions positively in cold tolerance by promoting polyamine accumulation by interacting with $\mathrm{ADC}$, which also provides a reference for improving cold tolerance of tea tree.

Author Contributions: X.Z. (Xujun Zhu), W.F. designed the manuscript, X.Z. (Xujun Zhu), X.Z. (Xue Zhao), T.R. conducted the experiments, X.Z. (Xue Zhao), Y.M., Y.W. analyzed the data, X.Z. (Xujun Zhu) drafted the manuscript, X.Z. (Xujun Zhu), W.F. supervised the project. All authors have read and approved the final version of this manuscript.

Funding: This research was supported by The National Natural Science Foundation of China (31870680), the earmarked fund for China Agriculture Research System (CARS-19), Jiangsu Agricultural Industry Technology System (JATS(2019)423), Keypoint Research and Invention Program of Jiangsu Province (BE2016417); (BE2019379), and Nanjing Agricultural Science and Technology Industry-University-Research Project (2019RHJD203). 
Conflicts of Interest: The authors declare no conflict of interest.

\section{References}

1. Thomashow, M.F. Plant cold acclimation: Freezing tolerance genes and regulatory mechanisms. Annu. Rev. Plant Biol. 1999, 50, 571-599. [CrossRef] [PubMed]

2. Hoard, K.G.; Crosbie, T.M. Correlated changes in agronomic traits from S1-line recurrent selection for cold tolerance in two maize populations. Crop Sci. 1986, 26, 519-522. [CrossRef]

3. Nakashima, K.; Ito, Y.; Yamaguchi-Shinozaki, K. Transcriptional regulatory networks in response to abiotic stresses in Arabidopsis and grasses. Plant Physiol. 2009, 149, 88-95. [CrossRef] [PubMed]

4. Chinnusamy, V.; Zhu, J.; Zhu, J.K. Gene regulation during cold acclimation in plants. Physiol. Plant. 2006, 126, 52-61. [CrossRef]

5. Medina, J.; Catala, R.; Salinas, J. The CBFs, three Arabidopsis transcription factors to cold acclimate. Plant Sci. 2011, 180, 3-11. [CrossRef]

6. Liu, J.H.; Peng, T.; Dai, W.S. Critical cis-acting elements and interacting transcription factors, key players associated with abiotic stress responses in plants. Plant Mol. Biol. Rep. 2014, 32, 303-317. [CrossRef]

7. Chinnusamy, V.; Zhu, J.K.; Sunkar, R. Gene regulation during cold stress acclimation in plants. Methods Mol. Biol. 2010, 639, 39-55. [CrossRef]

8. Lee, B.H.; Henderson, D.A.; Zhu, J.K. The Arabidopsis cold responsive transcriptome and its regulation by ICE1. Plant Cell 2005, 17, 3155-3175. [CrossRef]

9. Chinnusamy, V.; Zhu, J.; Zhu, J.K. Cold stress regulation of gene expression in plants. Trends Plant Sci. 2007, 12, 444-451. [CrossRef]

10. Chinnusamy, V.; Ohta, M.; Kanrar, S.; Lee, B.H.; Hong, X.H.; Agarwal, M.; Zhu, J.K. ICE1, a regulator of cold-induced transcriptome and freezing tolerance in Arabidopsis. Gene Dev. 2003, 17, 1043-1054. [CrossRef]

11. Feng, X.M.; Zhao, Q.; Zhao, L.L.; Qiao, Y.; Xie, X.B.; Li, H.F.; Yao, Y.X.; You, C.X.; Hao, Y.J. The cold-induced basic helix-loop-helix transcription factor gene $\mathrm{MdCIbHLH1}$ encodes an ICE-like protein in apple. BMC Plant Biol. 2012, 12, 22. [CrossRef] [PubMed]

12. Feng, H.L.; Ma, N.N.; Meng, X.; Zhang, S.; Wang, J.R.; Chai, S.; Meng, Q.W. A novel tomato MYC-type ICE1-like transcription factor, SIICE1a, confers cold, osmotic and salt tolerance in transgenic tobacco. Plant Physiol. Biochem. 2013, 73, 309-322. [CrossRef] [PubMed]

13. Huang, X.S.; Wang, W.; Zhang, Q.; Liu, J.H. A basic helix-loop-helix transcription factor, PtrbHLH, of Poncirus trifoliata confers cold tolerance and modulates peroxidase-mediated scavenging of hydrogen peroxide. Plant Physiol. 2013, 162, 1178-1194. [CrossRef] [PubMed]

14. Shan, W.; Kuang, J.F.; Lu, W.J.; Chen, J.Y. Banana fruit NAC transcription factor MaNAC1 is a direct target of MaICE1 and involved in cold stress through interacting with MaCBF1. Plant Cell Environ. 2014, 37, $2116-2127$. [CrossRef] [PubMed]

15. Li, X.W.; Feng, Z.G.; Yang, H.M.; Zhu, X.P.; Liu, J.; Yuan, H.Y. A novel cold-regulated gene from Camellia sinensis, CsCOR1, enhances salt- and dehydration-tolerance in tobacco. Biochem. Biophys. Res. Commun. 2010, 394, 354-359. [CrossRef] [PubMed]

16. Wang, L.; Li, X.W.; Zhao, Q.; Jing, S.L.; Chen, S.F.; Yuan, H.Y. Identification of genes induced in response to low-temperature treatment in tea leaves. Plant Mol. Biol. Rep. 2009, 27, 257-265. [CrossRef]

17. Wang, L.; Cao, H.; Qian, W.; Yao, L.; Hao, X.; Li, N.; Yang, Y.; Wang, X. Identification of a novel bZIP transcription factor in Camellia sinensis as a negative regulator of freezing tolerance in transgenic arabidopsis. Ann. Bot. 2017, 119, 1195-1209. [CrossRef]

18. Zhu, X.; Li, Q.; Hu, J.; Wang, M.; Li, X. Molecular cloning and characterization of spermine synthesis gene associated with cold tolerance in tea plant (Camellia sinensis). Appl. Biochem. Biotechnol. 2015, 177, 1055-1068. [CrossRef]

19. Wang, Y.; Jiang, C.J.; Li, Y.Y.; Wei, C.L.; Deng, W.W. CsICE1 and CsCBF1: Two transcription factors involved in cold responses in Camellia sinensis. Plant Cell Rep. 2012, 31, 27-34. [CrossRef]

20. Ding, Z.T.; Li, C.; Shi, H.; Wang, H.; Wang, Y. Pattern of CsICE1 expression under cold or drought treatment and functional verification through analysis of transgenic Arabidopsis. Genet. Mol. Res. 2015, 14, 11259-11270. [CrossRef] 
21. Zhu, X.J.; Thalor, S.K.; Takahashi, Y.; Berberich, T.; Kusano, T. An inhibitory effect of the sequence-conserved upstream open-reading frame on the translation of the main open-reading frame of HsfB1 transcripts in Arabidopsis. Plant Cell Environ. 2012, 35, 2014-2030. [CrossRef] [PubMed]

22. Clough, S.J.; Bent, A.F. Floral dip: A simplified method for Agrobacterium-mediated transformation of Arabidopsis thaliana. Plant J. 1998, 16, 735-743. [CrossRef] [PubMed]

23. Zhang, R.H.; Li, J.; Guo, S.R.; Tezuka, T. Effects of exogenous putrescine on gas-exchange characteristics and chlorophyll fluorescence of NaCl-stressed cucumber seedlings. Photosynth. Res. 2009, 100, 155-162. [CrossRef] [PubMed]

24. Xu, Q.; Shi, C.; Wan, X.; Tang, Z. Cloning and sequence analysis of arginine decarboxylase gene from Camellia sinensis. J. Anhui Agricul. Univ. 2013, 40, 464-469. (In Chinese)

25. Chen, H.C.; Hsieh-Feng, V.; Liao, P.C.; Cheng, W.H.; Liu, L.Y.; Yang, Y.W.; Lai, M.H.; Chang, M.C. The function of OsbHLH068 is partially redundant with its homolog, AtbHLH112, in the regulation of the salt stress response but has opposite functions to control flowering in Arabidopsis. Plant Mol. Biol. 2017, 94, 531-548. [CrossRef] [PubMed]

26. Le Hir, R.; Castelain, M.; Chakraborti, D.; Moritz, T.; Dinant, S.; Bellini, C. AtbHLH68 transcription factor contributes to the regulation of ABA homeostasis and drought stress tolerance in Arabidopsis thaliana. Physiol. Plant. 2017, 160, 312-327. [CrossRef] [PubMed]

27. Toledo-Ortiz, G.; Huq, E.; Quail, P.H. The Arabidopsis basic/helixloop-helix transcription factor family. Plant Cell 2003, 15, 1749-1770. [CrossRef]

28. Cuevas, J.C.; Lopez-Cobollo, R.; Alcazar, R.; Zarza, X.; Koncz, C.; Altabella, T.; Salinas, J.; Tiburcio, A.F.; Ferrando, A. Putrescine is involved in Arabidopsis freezing tolerance and cold acclimation by regulating abscisic acid levels in response to low temperature. Plant Physiol. 2008, 148, 1094-1105. [CrossRef]

29. Kou, S.; Chen, L.; Tu, W.; Scossa, F.; Wang, Y.; Liu, J.; Fernie, A.R.; Song, B.; Xie, C. The arginine decarboxylase gene $A D C 1$, associated to the putrescine pathway, plays an important role in potato cold-acclimated freezing tolerance as revealed by transcriptome and metabolome analyses. Plant J. 2018, 96, 1283-1298. [CrossRef]

30. Wang, J.; Sun, P.P.; Chen, C.L.; Wang, Y.; Fu, X.Z.; Liu, J.H. An arginine decarboxylase gene PtADC from Poncirus trifoliata confers abiotic stress tolerance and promotes primary root growth in Arabidopsis. J. Exp. Bot. 2011, 62, 2899-2914. [CrossRef] 